\title{
A New Second-Order Difference Approximation for Nonlocal Boundary Value Problem with Boundary Layers
}

\section{Derya Arslan}

\author{
University of Bitlis Eren \\ Department of Mathematics, Faculty of Art and Science, 13200, Bitlis, \\ Turkey \\ E-mail(corresp.): ayredlanu@gmail.com
}

Received April 2, 2019; revised January 31, 2020; accepted January 31, 2020

\begin{abstract}
The aim of this paper is to present finite difference method for numerical solution of singularly perturbed linear differential equation with nonlocal boundary condition. Initially, the nature of the solution of the presented problem for the numerical solution is discussed. Subsequently, the difference scheme is established on Bakhvalov-Shishkin mesh. Uniform convergence in the second-order is proven with respect to the $\varepsilon$ - perturbation parameter in the discrete maximum norm. Finally, an example is provided to demonstrate the success of the presented numerical method. Thus, it is shown that indicated numerical results support theoretical results.
\end{abstract}

Keywords: singular perturbation, finite difference method, Bakhvalov-Shishkin mesh, uniformly convergence, nonlocal condition.

AMS Subject Classification: 65L10; 65L11; 65L12; 65L15; 65L20; 65L70; 34B10.

\section{Introduction}

In the present study, linear singularly perturbed problem with nonlocal boundary condition is discussed as follows:

$$
\begin{aligned}
& \varepsilon^{2} u^{\prime \prime}(x)+\varepsilon a(x) u^{\prime}(x)-b(x) u(x)=f(x), \quad 0<x<1, \\
& u(0)=A \\
& u(1)-\gamma u\left(l_{1}\right)=B, \quad 0<l_{1}<1,
\end{aligned}
$$

where $0<\varepsilon<<1$ is a small perturbation parameter; $A, B$ and $|\gamma|<1$ are given constants; $a(x) \geq 0, b(x) \geq \beta>0$ and $f(x)$ are assumed to be sufficiently 
smooth functions in $[0,1]$. Furthermore, the solution of the problem (1.1)-(1.3) is within general boundary layers at $x=0$ and $x=1$ points.

Problems such as the nonlocal singular perturbation problem (1.1)-(1.3), are problems where the coefficients of the highest order derivative are a very small positive parameters such as $0<\varepsilon<<1$. Solving this problems with classical numerical methods may not be the right choice due to the difficulties that may arise from the small perturbation parameter [18, 23, 24, 25, 26, 27]. These difficulties are quick and fairly irregular variations within thin transition layers. These lead to unlimited number of derivatives in the solution of singular perturbation problems. Therefore, it is important to choose the most suitable numerical methods for singularly perturbed problems. These include finite difference and finite element methods. Thus, in the present study, we wanted to demonstrate that these difficulties can be overcome with the finite difference method.

Studies conducted on singular perturbation problems commenced in the 1900s. These problems were known to be common in the fields of natural sciences, engineering, medical sciences, fluid mechanics, aerodynamics, magnetic dynamics, diffusion theory, reaction diffusion, light emitting waves, electron plasma waves, communication networks, plasma dynamics, refined gas dynamics, mass transport, plastics, chemical reactor theory, oceanography, meteorology, electricity current, ion acoustic waves plasma and several physical modelling techniques (see, $[2,4,9,14,15,18,24,25,26,27]$ ). Lately, singularly perturbed problems, particularly with the nonlocal boundary condition and boundary layers have been studied by several researchers (e.g., [1,7,8,10,11,12, $16,17,19,20,23]$ and the references therein). Bakhvalov used a special transformation in numerical solution of boundary solid problems [5]. Bitsadze and Samarskii obtained several generalizations for linear elliptic boundary value problems [6]. Čiegis, studied numerical solution of the singular perturbation problem with nonlinear boundary condition [13]. Different from the previous studies in the literature and for the first time, this problem is solved with the presented finite difference method on Bakhvalov-Shishkin mesh in order to demonstrate that the difference scheme has second-order convergence and a better result could be obtained. Especially, this method shows uniformly convergent provided only that $\varepsilon \leq C N^{-1}$. Namely, Bakhvalov-Shishkin mesh gives a stronger error bound for $\varepsilon \leq C N^{-1}$. Bakhvalov-Shishkin mesh is a modification of the Shishkin mesh described that incorporates idea by Bakhvalov. But the original Bakhvalov mesh requires the solution of a nonlinear equation to determine the transition point where the mesh switches from coarse to fine. Instead, the transition points are as in the Shishkin mesh [21]. There are many studies on the B-S (Bakhvalov-Shishkin) mesh: T. Linss has studied simple upwind difference scheme on a B-S mesh [21]. Analysis of a Galerkin finite element method on Bakhvalov-Shishkin mesh for a linear convection-diffusion problem is investiagated by Linss [22]. Uniform second-order hybrid schemes on Bakhvalov-Shishkin mesh are analyzed in [29]. Hybrid difference schemes with variable weights on Bakhvalov-Shishkin mesh are examined to the derivative for quasi-linear singularly perturbed convection-diffusion boundary value problems in [28]. Linear Galerkin finite element method on Bakhvalov-Shishkin 
mesh for singularly perturbed convection-diffusion problem is worked in [30].

The present study is structured as follows: Section 2 focused on the exact solution of the problem provided in (1.1)-(1.3) and several asymptotic evaluations on the fourth-order derivatives of the exact solution. In Section 3, the difference scheme is constructed as hybrid scheme. Subsequently, the structure of the Bakhvalov-Shishkin mesh is introduced. In Section 4, the second-order uniform convergence of the difference scheme is obtained according to $\varepsilon$. The present study is finalized with the conclusion section. Henceforth, $C$ and $C_{0}$ are positive constants independent of $\varepsilon$ and the mesh parameter in the following sections.

\section{Certain properties of the continuous problem}

This section focuses on some properties of the solution $u(x)$ of the problem (1.1)-(1.3), which will be essential in the further sections of the study.

Lemma 1. Given that $a(x), b(x)$ and $f(x) \in C^{3}[0,1]$. Then, the solution of the problem (1.1)-(1.3) fulfills the following inequalities:

$$
\begin{aligned}
& |u(x)| \leq C_{0}, \\
& \left|u^{(k)}(x)\right| \leq C\left\{1+\frac{1}{\varepsilon^{k}}\left(e^{-\frac{\mu_{1} x}{\varepsilon}}+e^{-\frac{\mu_{2}(1-x)}{\varepsilon}}\right)\right\}, \quad 0<x<1, \quad k=1,2,3,
\end{aligned}
$$

where

$$
\begin{aligned}
& C_{0}=|A|+(1-|\gamma|)^{-1}\left[|B|+|\gamma|\left(|A|+\beta^{-1}\|f\|_{\infty}\right)\right]+\beta^{-1}\|f\|_{\infty},|\gamma|<1, \\
& \mu_{1}=\frac{1}{2}\left(\sqrt{a^{2}(0)+4 b(0)}+a(0)\right), \quad \mu_{2}=\frac{1}{2}\left(\sqrt{a^{2}(1)+4 b(0)}-a(1)\right) .
\end{aligned}
$$

Proof. Once maximum principle for (1.1) is used, we obtain that

$$
|u(x)| \leq|A|+|u(1)|+\beta^{-1}\|f\|_{\infty} .
$$

Next, from boundary condition (1.3), we attain

$$
|u(1)| \leq|B|+|\gamma|\left|u\left(l_{1}\right)\right| .
$$

If $x=l_{1}$ is written in inequality (2.3), the following inequality is found

$$
\left|u\left(l_{1}\right)\right| \leq|A|+|u(1)|+\beta^{-1}\|f\|_{\infty} .
$$

By setting (2.5) in inequality (2.4), we get

$$
|u(1)| \leq(1-|\gamma|)^{-1}\left[|B|+|\gamma|\left(|A|+\beta^{-1}\|f\|_{\infty}\right)\right] .
$$

Then by setting (2.6) in inequality (2.3), we have

$$
|u(x)| \leq|A|+(1-|\gamma|)^{-1}\left[|B|+|\gamma|\left(|A|+\beta^{-1}\|f\|_{\infty}\right)\right]+\beta^{-1}\|f\|_{\infty},
$$

where

$$
C_{0}=|A|+(1-|\gamma|)^{-1}\left[|B|+|\gamma|\left(|A|+\beta^{-1}\|f\|_{\infty}\right)\right]+\beta^{-1}\|f\|_{\infty},
$$


and this prove the inequality (2.1).

The proof of inequality (2.2) is almost identical to that of $[1,9]$ for $k=1$ as

$$
\left|u^{\prime}(x)\right| \leq C\left\{1+\frac{1}{\varepsilon}\left(e^{-\frac{\mu_{1} x}{\varepsilon}}+e^{-\frac{\mu_{2}(1-x)}{\varepsilon}}\right)\right\}, \quad 0<x<1, \quad k=1 .
$$

Now, we obtain inequality (2.2) for $k=2$. The proof of (2.2) for $k=3$ is obtained in the same way. Let us begin by taking the derivative of equation (1.1) two times,

$$
\begin{aligned}
& \varepsilon^{2} v^{\prime \prime}(x)+\varepsilon a(x) v^{\prime}(x)-b(x) v(x)=G(x), \\
& v(0)=u^{\prime \prime}(0), \quad v(1)=u^{\prime \prime}(1),
\end{aligned}
$$

where

$$
\begin{aligned}
& u^{\prime \prime}(x)=v(x), \\
& G(x)=f^{\prime \prime}(x)-2 \varepsilon a^{\prime}(x) u^{\prime \prime}(x)-\left(\varepsilon a^{\prime \prime}(x)-2 b^{\prime}\right) u^{\prime}+b^{\prime \prime}(x) u(x),
\end{aligned}
$$

and also, from (1.1) we get

$$
u^{\prime \prime}(0) \leq \frac{C}{\varepsilon^{2}}, \quad u^{\prime \prime}(1) \leq \frac{C}{\varepsilon^{2}} .
$$

Now, let us take $v(x)$ as follows:

$$
v(x)=v_{1}(x)+v_{2}(x),
$$

where $v_{1}(x)$ and $v_{2}(x)$ are the solutions of the following problems:

$$
\begin{array}{ll}
L v_{1}(x)=G(x), \quad v_{1}(0)=0, & v_{1}(1)=0, \\
L v_{2}(x)=0, \quad v_{1}(0)=u^{\prime \prime}(0), & v_{1}(1)=u^{\prime \prime}(1),
\end{array}
$$

From (2.8), (2.10) and [1], we have

$$
\left|v_{1}(x)\right| \leq C .
$$

We can give the solution of (2.11) in the form

$$
v_{2}(x)=p_{0}(x)+q_{0}(x)+R_{\varepsilon}(x),
$$

where the functions $p_{0}(x), q_{0}(x)$ and $R_{\varepsilon}(x)$ are, respectively, the solutions of (2.21), (2.22) and (2.23) from [1]. Also, we see that these solutions have the following estimations:

$$
\left|p_{0}^{(m)}(x)\right| \leq \frac{C}{\varepsilon^{m+1}} e^{-\frac{\mu_{1} x}{\varepsilon}},\left|q_{0}^{(m)}(x)\right| \leq \frac{C}{\varepsilon^{m+1}} e^{-\frac{\mu_{2}(1-x)}{\varepsilon}}, \quad m=0,1,2,
$$

and $\left|R_{\varepsilon}(x)\right| \leq C$. From (2.7), (2.9), (2.12) and (2.13) the following inequality clearly leads to $(2.2)$ for $k=2$.

$$
\begin{gathered}
\left|u^{\prime \prime}(x)\right|=|v(x)| \leq\left|v_{1}(x)\right|+\left|v_{2}(x)\right| \leq\left|p_{0}(x)\right|+\left|q_{0}(x)\right|+\left|R_{\varepsilon}(x)\right| \\
\leq \frac{C}{\varepsilon} e^{-\frac{\mu_{1} x}{\varepsilon}}+\frac{C}{\varepsilon} e^{-\frac{\mu_{2}(1-x)}{\varepsilon}}+C \leq \frac{C}{\varepsilon^{2}} e^{-\frac{\mu_{1} x}{\varepsilon}}+\frac{C}{\varepsilon^{2}} e^{-\frac{\mu_{2}(1-x)}{\varepsilon}}+C \\
\leq C\left\{1+\frac{1}{\varepsilon^{2}}\left(e^{-\frac{\mu_{1} x}{\varepsilon}}+e^{-\frac{\mu_{2}(1-x)}{\varepsilon}}\right)\right\}, \quad 0<x<1, \quad k=2 .
\end{gathered}
$$

All these estimations conclude our proof. 


\section{The construction of difference scheme and mesh}

In this section, the discretization of the problem (1.1)-(1.3) using finite difference method on Bakhvalov-Shishkin mesh is presented.

\subsection{Bakhvalov-Shishkin mesh}

The interval $[0,1]$ is divided into the three subintervals $\left[0, \sigma_{1}\right],\left[\sigma_{1}, 1-\sigma_{2}\right]$ and $\left[1-\sigma_{2}, 1\right]$. Here $\sigma_{1}$ and $\sigma_{2}$ are referred as the transition points and are written as follows:

$$
\sigma_{1}=\min \left\{\frac{1}{4}, \mu_{1}^{-1} \varepsilon \ln N\right\}, \quad \sigma_{2}=\min \left\{\frac{1}{4}, \mu_{2}^{-1} \varepsilon \ln N\right\} .
$$

Assumption 1: We shall assume throughout the paper that $\varepsilon \leq C N^{-1}$ as is generally the case in practice, where, $N$ is a positive even integer.

The mesh points $\bar{\omega}_{N}=\left\{x_{i}\right\}_{i=0}^{N}$ are introduced through a set of the equalities:

$$
x_{i}=\left\{\begin{array}{c}
-\mu_{1}^{-1} \varepsilon \ln \left[1-4\left(1-N^{-1}\right) \frac{i}{N}\right], \quad i=0, \ldots, \frac{N}{4} \\
\sigma_{1}+\left(i-\frac{N}{4}\right) h, \quad h=\frac{2\left(1-\sigma_{2}-\sigma_{1}\right)}{N}, i=\frac{N}{4}+1, \ldots, \frac{3 N}{4} \\
1+\mu_{2}^{-1} \varepsilon \ln \left[1-4\left(1-N^{-1}\right)\left(1-\frac{i}{N}\right)\right], \quad i=\frac{3 N}{4}, \ldots, N
\end{array}\right.
$$

\subsection{The construction of the difference scheme}

Here the following finite differences for any mesh function $g_{i}=g\left(x_{i}\right)$ are presented on $\bar{\omega}_{N}$ as:

$$
\begin{aligned}
& g_{\bar{x}, i}=\frac{g_{i}-g_{i-1}}{h_{i}}, \quad g_{x, i}=\frac{g_{i+1}-g_{i}}{h_{i+1}}, \quad g_{0, i}=\frac{g_{x, i}+g_{\bar{x}, i}}{2}, \\
& g_{\widehat{x}, i}=\frac{g_{i+1}-g_{i}}{\hbar_{i}}, \quad g_{\bar{x} \widehat{x}, i}=\frac{g_{x, i}-g_{\bar{x}, i}}{\hbar_{i}}, \quad \hbar_{i}=\frac{h_{i}+h_{i+1}}{2}, \quad h_{i}=x_{i}-x_{i-1}, \\
& \|g\|_{\infty} \equiv\|g\|_{\infty, \bar{\omega}_{N}}:=\max _{0 \leqslant i \leqslant N}\left|g_{i}\right| .
\end{aligned}
$$

Now, the difference scheme for the problem (1.1) should be constructed.

The following exact relation is obtained through the use of the interpolating quadrature formulas on subintervals $\left[x_{i-1}, x_{i+1}\right]$ [2]. Initially, the equation (1.1) is integrated over $\left(x_{i-1}, x_{i+1}\right)$ as

$$
\hbar_{i}^{-1} \int_{x_{i-1}}^{x_{i+1}} L u(x) \varphi_{i}(x) d x=\hbar_{i}^{-1} \int_{x_{i-1}}^{x_{i+1}} f(x) \varphi_{i}(x) d x, i=1, \ldots, \frac{N}{4}, i=\frac{3 N}{4}, \ldots, N-1 .
$$

If the above equality is arranged, it gives

$$
\hbar_{i}^{-1} \int_{x_{i-1}}^{x_{i+1}}\left[\varepsilon^{2} u^{\prime \prime(x)}+\varepsilon a(x) u^{\prime}(x)-b(x) u(x)\right] \varphi_{i}(x) d x=\hbar_{i}^{-1} \int_{x_{i-1}}^{x_{i+1}} f(x) \varphi_{i}(x) d x .
$$

From here, the following equality is obtained by implementing partial integration and then by using the formula $(2.2)$ of [3]:

$$
\hbar_{i}^{-1} \varepsilon^{2} \int_{x_{i-1}}^{x_{i+1}} u^{\prime}(x) \varphi_{i}^{\prime}(x) d x+\hbar_{i}^{-1} \varepsilon a_{i} \int_{x_{i-1}}^{x_{i+1}} u^{\prime}(x) \varphi_{i}(x) d x-b_{i} u_{i}=f_{i}-R_{i}^{1} .
$$


Finally, we propose the following difference scheme for approximating (1.1)$(1.3)$ :

$$
\varepsilon^{2} u_{\bar{x} \widehat{x}, i}+\varepsilon a_{i} u_{x, i}-b_{i} u_{i}=f_{i}-R_{i}^{1}, i=1, \ldots, \frac{N}{4}, i=\frac{3 N}{4}, \ldots, N-1,
$$

and the reminder term

$$
R_{i}^{1}=-\frac{\varepsilon^{2}}{2} \int_{x_{i-1}}^{x_{i+1}} \varphi_{i}(x) u^{\prime \prime \prime}(x) d x-\frac{\varepsilon a_{i}}{h_{i+1}} \int_{x_{i}}^{x_{i+1}}\left(x_{i+1}-x\right) u^{\prime \prime}(x) d x,
$$

where the functions $\varphi_{i}(x)$ are in the form:

$$
\varphi_{i}(x)= \begin{cases}\frac{\left(x-x_{i-1}\right)^{2}}{\hbar_{i} h_{i}}, & x_{i-1}<x<x_{i} \\ \frac{\left(x_{i+1}-x\right)^{2}}{\hbar_{i} h_{i+1}}, & x_{i}<x<x_{i+1} .\end{cases}
$$

Secondly, Equation (1.1) is integrated over $\left(x_{i-1}, x_{i+1}\right)$ as

$$
\begin{gathered}
h^{-1} \int_{x_{i-1}}^{x_{i+1}} L u(x) \psi_{i}(x) d x=h^{-1} \int_{x_{i-1}}^{x_{i+1}} f(x) \psi_{i}(x) d x, \quad i=\frac{N}{4}+1, \ldots, \frac{3 N}{4}-1, \\
h^{-1} \int_{x_{i-1}}^{x_{i+1}}\left[\varepsilon^{2} u^{\prime \prime}(x)+\varepsilon a(x) u^{\prime}(x)-b(x) u(x)\right] \psi_{i}(x) d x=h^{-1} \int_{x_{i-1}}^{x_{i+1}} f(x) \psi_{i}(x) d x .
\end{gathered}
$$

Here, applying partial integration in the first expression of the left integral, we get

$$
h^{-1} \varepsilon^{2} \int_{x_{i-1}}^{x_{i+1}} u^{\prime}(x) \psi_{i}^{\prime}(x) d x+h^{-1} \varepsilon a_{i} \int_{x_{i-1}}^{x_{i+1}} u^{\prime}(x) \psi_{i}(x) d x-b_{i} u_{i}=f_{i}-R_{i}^{2},
$$

and from here it follows that

$$
\begin{aligned}
& h^{-1} \int_{x_{i-1}}^{x_{i}}\left(\varepsilon^{2} u^{\prime}(x) \psi_{i}^{\prime}(x)+\varepsilon a_{i} u^{\prime}(x) \psi_{i}(x)\right) d x \\
& \quad+\int_{x_{i}}^{x_{i+1}}\left(\varepsilon^{2} u^{\prime}(x) \psi_{i}^{\prime}(x)+\varepsilon a_{i} u^{\prime}(x) \psi_{i}(x)\right) d x=b_{i} u_{i}+f_{i}-R_{i}^{2},
\end{aligned}
$$

we use the formula (2.2) of [3] in (3.2) and propose the following difference scheme:

$$
\varepsilon^{2} u_{\bar{x} x, i}+\varepsilon a_{i} u_{x, i}-b_{i} u_{i}=f_{i}-R_{i}^{2}, \quad i=\frac{N}{4}+1, \ldots, \frac{3 N}{4}-1,
$$

where reminder term $R_{i}^{2}$

$$
R_{i}^{2}=-\frac{\varepsilon^{2}}{2} \int_{x_{i-1}}^{x_{i+1}} \psi_{i}(x) u^{\prime \prime \prime}(x) d x-\frac{\varepsilon a_{i}}{h} \int_{x_{i}}^{x_{i+1}}\left(x_{i+1}-x\right) u^{\prime \prime}(x) d x,
$$


and the functions $\psi_{i}(x)$ take the form

$$
\psi_{i}(x)=\left\{\begin{array}{l}
-\left(\frac{x-x_{i-1}}{h}\right)^{2}, \quad x_{i-1}<x<x_{i}, \\
\left(\frac{x_{i+1}-x}{h}\right)^{2}, \quad x_{i}<x<x_{i+1} .
\end{array}\right.
$$

Here, it is necessary to define an approximation for the second boundary condition (1.3). The following equation is written using the interpolation quadrature formula with respect to $x_{N_{0}}$ and $x_{N_{0}+1}$ :

$$
u(x)=\frac{x-x_{N_{0}+1}}{x_{N_{0}}-x_{N_{0}+1}} u\left(x_{N_{0}}\right)+\frac{x-x_{N_{0}}}{x_{N_{0}+1}-x_{N_{0}}} u\left(x_{N_{0}+1}\right)+r_{0},
$$

where, reminder term $r_{0}$

$$
r_{0}=\frac{1}{2} f^{\prime \prime}(\xi)\left(x-x_{N_{0}}\right)\left(x-x_{N_{0}+1}\right), \quad \xi \in\left(x_{N_{0}}, l_{1}\right) .
$$

Once reminder terms $R_{i}^{1}$ and $R_{i}^{2}$ and $r_{0}$ are neglected from (3.1), (3.3) and (3.4), it is possible to propose the following difference schemes for the problem (1.1)-(1.3):

$$
\begin{aligned}
& \varepsilon^{2} y_{\bar{x} \widehat{x}, i}+\varepsilon a_{i} y_{x, i}-b_{i} y_{i}=f_{i}, i=1, \ldots, \frac{N}{4}, i=\frac{3 N}{4}, \ldots N, \\
& \varepsilon^{2} y_{\bar{x} x, i}+\varepsilon a_{i} y_{x, i}-b_{i} y_{i}=f_{i}, i=\frac{N}{4}+1, \ldots, \frac{3 N}{4}-1, \\
& y_{0}=A \\
& y_{N}-\gamma\left[\frac{l_{1}-x_{N_{0}+1}}{x_{N_{0}}-x_{N_{0}+1}} y\left(x_{N_{0}}\right)+\frac{l_{1}-x_{N_{0}}}{x_{N_{0}+1}-x_{N_{0}}} y\left(x_{N_{0}+1}\right)\right]=B,
\end{aligned}
$$

where $x_{N_{0}}$ is the mesh point nearest to $l_{1}$.

\section{Uniform error estimates}

With respect to the examination of the presented method for the problem (1.1)(1.3), this section provides the following discrete problem and its solution:

$$
\begin{aligned}
& \varepsilon^{2} z_{\bar{x} \widehat{x}, i}+\varepsilon a_{i} z_{x, i}-b_{i} z_{i}=R_{i}^{1}, \quad i=0, \ldots, \frac{N}{4}, i=\frac{3 N}{4}, \ldots, N, \\
& \varepsilon^{2} z_{\bar{x} x, i}+\varepsilon a_{i} z_{x, i}-b_{i} z_{i}=R_{i}^{2}, i=\frac{N}{4}+1, \ldots, \frac{3 N}{4}-1, \\
& z_{0}=0, z_{N}-\gamma\left[\frac{l_{1}-x_{N_{0}+1}}{x_{N_{0}}-x_{N_{0}+1}} z\left(x_{N_{0}}\right)+\frac{l_{1}-x_{N_{0}}}{x_{N_{0}+1}-x_{N_{0}}} z\left(x_{N_{0}+1}\right)\right]=r_{0},
\end{aligned}
$$

where, $R_{i}^{1}, R_{i}^{2}$ and $r_{0}$ are given by (3.1), (3.3) and (3.4) respectively.

Lemma 2. If $z_{i}$ is the solution to (4.1)-(4.2), then the estimate becomes:

$$
\|z\|_{\infty, \bar{\omega}_{N}} \leq C\left(\left\|R^{1}\right\|_{\infty, \omega_{N}}+\left\|R^{2}\right\|_{\infty, \omega_{N}}+\left|r_{0}\right|\right)
$$

holds. 
Proof. Once the maximum principle is used for (4.1)-(4.2), we deduce

$$
\begin{aligned}
& \left|z_{i}\right| \leq\left|z_{N}\right|+\beta^{-1}\left[\left\|R^{1}\right\|_{\infty, \omega_{N}}+\left\|R^{2}\right\|_{\infty, \omega_{N}}\right] \\
& \left|z_{N}\right| \leq\left|r_{0}\right|+|\gamma|\left|z_{N_{0}}\right|
\end{aligned}
$$

where $|\gamma| \leq k<1$. For $i=N_{0}$ in the Equation (4.3), it yield:

$$
\left|z_{N_{0}}\right| \leq\left|z_{N}\right|+\beta^{-1}\left[\left\|R^{1}\right\|_{\infty, \omega_{N}}+\left\|R^{2}\right\|_{\infty, \omega_{N}}\right] .
$$

As a result, from (4.3)-(4.4), we obtain

$$
\begin{aligned}
\left|z_{i}\right| \leq & (1-k)^{-1}\left\{\left|r_{0}\right|+k \beta^{-1}\left[\left\|R^{1}\right\|_{\infty, \omega_{N}}+\left\|R^{2}\right\|_{\infty, \omega_{N}}\right]\right\} \\
& +\beta^{-1}\left[\left\|R^{1}\right\|_{\infty, \omega_{N}}+\left\|R^{2}\right\|_{\infty, \omega_{N}}\right] .
\end{aligned}
$$

And conclusively, from here, we obtain Lemma 2.

Lemma 3. Based on the assumptions of Lemma 1 and Lemma 2, the solution of the problem (1.1)-(1.3) fulfills the following estimates for the reminder terms $R_{i}^{1}, R_{i}^{2}$ and $r_{0}$ :

$$
\left\|R^{1}\right\|_{\infty, \omega_{N}} \leq C N^{-2}, \quad\left\|R^{2}\right\|_{\infty, \omega_{N}} \leq C N^{-2}, \quad\left|r_{0}\right| \leq C N^{-2} .
$$

Proof. The remainder terms $R_{i}^{1}, R_{i}^{2}$ and $r_{0}$ are evaluated for the subintervals $\left[0, \sigma_{1}\right],\left[\sigma_{1}, 1-\sigma_{2}\right]$ and $\left[1-\sigma_{2}, 1\right]$ on Bakhvalov-Shishkin mesh.

1) The remainder term $R_{i}^{1}$ is evaluated for $x_{i} \in\left[0, \sigma_{1}\right], \sigma_{1} \leq 1 / 4$ :

$$
\begin{aligned}
& x_{i-1}=-\mu_{1}^{-1} \varepsilon \ln \left[1-4\left(1-N^{-1}\right) \frac{(i-1)}{N}\right], i=1, \ldots, \frac{N}{4}, \\
& h_{i}=-\mu_{1}^{-1} \varepsilon \ln \left[1-4\left(1-N^{-1}\right) \frac{i}{N}\right]+\mu_{1}^{-1} \varepsilon \ln \left[1-4\left(1-N^{-1}\right) \frac{(i-1)}{N}\right] .
\end{aligned}
$$

Applying the mean value theorem in (4.6), we obtain that

$$
h_{i}=\mu_{1}^{-1} \varepsilon \frac{4\left(1-N^{-1}\right) N^{-1}}{1-4 i_{1}\left(1-N^{-1}\right) N^{-1}} \leq C N^{-1} .
$$

Thus, from (3.1) and (4.5)-(4.7), we can write

$$
\begin{aligned}
& \left|R_{i}^{1}\right| \leq C\left\{\varepsilon^{2} \int_{x_{i-1}}^{x_{i}}\left|u^{\prime \prime \prime}(x)\right| \frac{\left(x_{i-1}-x\right)^{2}}{\hbar_{i} h_{i}} d x\right\} \\
& +C\left\{\int_{x_{i}}^{x_{i+1}}\left[\varepsilon^{2}\left|u^{\prime \prime \prime}(x)\right| \frac{\left(x_{i+1}-x\right)^{2}}{\hbar_{i} h_{i+1}}+\varepsilon h_{i+1}^{-1}\left|u^{\prime \prime}(x)\right|\left(x_{i+1}-x\right)\right] d x\right\} \\
& \leq C\left\{\varepsilon^{2} \int_{x_{i-1}}^{x_{i+1}}\left|1+\frac{1}{\varepsilon^{3}}\left(e^{-\frac{\mu_{1}(x)}{\varepsilon}}+e^{-\frac{\mu_{2}(1-x)}{\varepsilon}}\right)\right| d x\right\} \\
& +C\left\{\varepsilon \int_{x_{i}}^{x_{i+1}}\left|1+\frac{1}{\varepsilon^{2}}\left(e^{-\frac{\mu_{1}(x)}{\varepsilon}}+e^{-\frac{\mu_{2}(1-x)}{\varepsilon}}\right)\right| d x\right\} .
\end{aligned}
$$


Since

$$
\begin{aligned}
e^{-\frac{\mu_{1}\left(x_{i-1}\right)}{\varepsilon}}-e^{-\frac{\mu_{1}\left(x_{i+1}\right)}{\varepsilon}} & =e^{\ln \left[1-\left(1-N^{-1}\right) \frac{4(i-1)}{N}\right]}-e^{\ln \left[1-\left(1-N^{-1}\right) \frac{4(i+1)}{N}\right]} \\
& \leq 8\left(1-N^{-1}\right) N^{-1} \leq C N^{-2}
\end{aligned}
$$

the following estimations are obtained in a similar manner:

$$
\begin{aligned}
& e^{-\frac{\mu_{2}\left(1-x_{i+1}\right)}{\varepsilon}}-e^{-\frac{\mu_{2}\left(1-x_{i-1}\right)}{\varepsilon}} \leq C N^{-2}, \\
& e^{-\frac{\mu_{1}\left(x_{i}\right)}{\varepsilon}}-e^{-\frac{\mu_{1}\left(x_{i+1}\right)}{\varepsilon}} \leq C N^{-2}, \quad e^{-\frac{\mu_{2}\left(1-x_{i+1}\right)}{\varepsilon}}-e^{-\frac{\mu_{2}\left(1-x_{i}\right)}{\varepsilon}} \leq C N^{-2} .
\end{aligned}
$$

It then follows from (4.8), we come to conclusion as

$$
\left|R_{i}^{1}\right| \leq C N^{-2}, \quad i=1, \ldots, N / 4 .
$$

2) The remainder term $R_{i}^{2}$ is evaluated for $x_{i} \in\left[\sigma_{1}, 1-\sigma_{2}\right]$ :

$$
x_{i}=\sigma_{1}+(i-N / 4) h, \quad i=N / 4+1, \ldots, 3 N / 4,
$$

where

$$
h=2\left(1-\sigma_{2}-\sigma_{1}\right) / N .
$$

It then follows from $(3.3),(4.9)$ and $(4.10)$, we have

$$
\begin{aligned}
\left|R_{i}^{2}\right| & \leq C\left\{\varepsilon^{2} \int_{x_{i-1}}^{x_{i+1}}\left|u^{\prime \prime \prime}(x)\right| d x+\varepsilon \int_{x_{i}}^{x_{i+1}}\left|u^{\prime \prime}(x)\right| d x\right\} \\
& \leq C\left\{\mu_{1}^{-1}\left[e^{\frac{-\mu_{1} x_{i-1}}{\varepsilon}}-e^{\frac{-\mu_{1} x_{i+1}}{\varepsilon}}\right]-\mu_{2}^{-1}\left[e^{\frac{-\mu_{2}\left(1-x_{i+1}\right)}{\varepsilon}}-e^{\left.\frac{-\mu_{2}\left(1-x_{i-1}\right)}{\varepsilon}\right]}\right]\right. \\
& \leq C N^{-2}, \quad i=N / 4+1, \ldots, 3 N / 4-1,
\end{aligned}
$$

where

$$
e^{-\frac{\mu_{1}\left(x_{i-1}\right)}{\varepsilon}}-e^{-\frac{\mu_{1}\left(x_{i+1}\right)}{\varepsilon}} \leq \frac{1}{N^{2}} e^{\frac{-\mu_{1}\left(i-1-\frac{N}{4}\right) h}{\varepsilon}}\left(1-e^{\frac{-2 \mu_{1} h}{\varepsilon}}\right) \leq C N^{-2} .
$$

and similarly

$$
e^{-\frac{\mu_{2}\left(1-x_{i+1}\right)}{\varepsilon}}-e^{-\frac{\mu_{2}\left(1-x_{i-1}\right)}{\varepsilon}} \leq C N^{-2}
$$

3) The remainder term $R_{i}^{1}$ is evaluated for $x_{i} \in\left[1-\sigma_{2}, 1\right]$ :

$$
\begin{aligned}
& x_{i-1}=1+\mu_{2}^{-1} \varepsilon \ln \left[1-4\left(1-N^{-1}\right)\left(1-\frac{i-1}{N}\right)\right], \quad i=\frac{3 N}{4}, \ldots, N \\
& h_{i}=\mu_{2}^{-1} \varepsilon\left\{\ln \left[1-4\left(1-N^{-1}\right)\left(1-\frac{i}{N}\right)\right]-\ln \left[1-4\left(1-N^{-1}\right)\left(1-\frac{i-1}{N}\right)\right]\right\} .
\end{aligned}
$$

By applying the mean value theorem in (4.12), we obtain

$$
h_{i} \leq C N^{-1} \text {. }
$$


Using the inequality (4.13), we have

$$
\hbar_{i} \leq C N^{-1}
$$

Thus, from (3.1) and (4.11)-(4.14), we can write

$$
\left|R_{i}^{1}\right| \leq C N^{-2}, \quad i=3 N / 4, \ldots, N
$$

where

$$
\begin{aligned}
e^{-\frac{\mu_{1}\left(x_{i-1}\right)}{\varepsilon}}-e^{-\frac{\mu_{1}\left(x_{i+1}\right)}{\varepsilon}}= & e^{-\mu_{1}\left(1+\mu_{2}^{-1} \varepsilon \ln \left[1-4\left(1-N^{-1}\right)\left(1-\frac{i-1}{N}\right)\right]\right)} \\
& -e^{-\mu_{1}\left(1+\mu_{2}^{-1} \varepsilon \ln \left[1-4\left(1-N^{-1}\right)\left(1-\frac{i+1}{N}\right]\right)\right.} \leq C N^{-2} .
\end{aligned}
$$

4) Now, we estimate the remainder term $r_{0}$. In the following estimation, $x_{N_{0}}$ is the mesh point nearest to $l_{1}$. Also, we assume that $l_{1} \in\left[2 \alpha^{-1} \varepsilon|\ln \varepsilon|\right.$, $\left.1-2 \alpha^{-1} \varepsilon|\ln \varepsilon|\right], \alpha \geq 0$, and the second derivative of $f(x)$ is bounded. So, we obtain from (3.4),

$$
\begin{aligned}
\left|r_{0}\right| & \leq C\left\{\left|f^{\prime \prime}(\xi)\left(x-x_{N_{0}}\right)\left(x-x_{N_{0}+1}\right)\right|\right\} \\
& \leq C\left\{\left(x-x_{N_{0}}\right)\left(x-x_{N_{0}+1}\right)\right\} \leq C\left\{h^{2}\right\} \leq C N^{-2}, \quad \xi \in\left(x_{N_{0}}, l_{1}\right) .
\end{aligned}
$$

These estimations complete the proof of Lemma 3.

We can state the convergence result of this study the following Theorem 1.

Theorem 1. Let $u(x)$ be the solution of the problem (1.1)-(1.3) and $y$ be the solution of (3.5)-(3.6). Then, the following uniform error estimate satisfies

$$
\|y-u\|_{\infty, \bar{\omega}_{N}} \leq C N^{-2} .
$$

Proof. This follows immediately by mixing previous lemmas.

\section{$5 \quad$ Algorithm and numerical results}

This section focuses on the demonstration of the following procedure for the difference scheme (3.5)-(3.6). Moreover, the effectiveness of the presented method is confirmed by applying it to a linear problem (1.1)-(1.3). Initially, the algorithm for the solution of the difference scheme (3.5)-(3.6) is provided:

$$
\begin{aligned}
& \left(\frac{\varepsilon^{2}}{\hbar h_{i}}\right) y_{i-1}-\left(\frac{\varepsilon^{2}}{\hbar h_{i+1}}+\frac{\varepsilon^{2}}{\hbar h_{i}}+\frac{\varepsilon a_{i}}{h_{i+1}}+b_{i}\right) y_{i}+\left(\frac{\varepsilon^{2}}{\hbar h_{i+1}}+\frac{\varepsilon a_{i}}{h_{i+1}}\right) y_{i+1}=-f_{i}, \\
& \quad i=1, \ldots, N / 4, \quad i=3 N / 4, \ldots, N-1 ; \\
& \left(\frac{\varepsilon^{2}}{h^{2}}\right) y_{i-1}-\left(\frac{2 \varepsilon^{2}}{h^{2}}+\frac{\varepsilon a_{i}}{h}+b_{i}\right) y_{i}+\left(\frac{\varepsilon^{2}}{h^{2}}+\frac{\varepsilon a_{i}}{h}\right) y_{i+1}=-f_{i}, i=\frac{N}{4}+1, \ldots, \frac{3 N}{4}-1 ; \\
& \alpha_{1}=0, \quad \beta_{1}=0, \\
& \alpha_{i+1}=\frac{B_{i}}{C_{i}-A_{i} \alpha_{i}}, \beta_{i+1}=\frac{F_{i}+A_{i} \beta_{i}}{C_{i}-A_{i} \alpha_{i}}, \quad i=1, \ldots, N-1, \\
& y_{i}=\alpha_{i+1} y_{i+1}+\beta_{i+1}, \quad i=N-1, \ldots, 1 .
\end{aligned}
$$


This algorithm is stable due to $A_{i}>0, B_{i}>0, C_{i}>A_{i}+B_{i}, i=1,2, \ldots, N$.

Subsequently, the following problem is taken into consideration in order to prove that the presented method is working:

$$
\begin{aligned}
& \varepsilon^{2} u^{\prime \prime}(x)+\varepsilon(1+\cos (\pi x)) u^{\prime}(x)-(1+\sin (\pi x / 2)) u(x)=f(x), \quad 0<x<1, \\
& u^{\prime}(0)=0, \quad u(1)-0.5 u(0.5)=0.5 .
\end{aligned}
$$

The exact solution of the problem is

$$
u(x)=\frac{\frac{\left(1-e^{1-x}\right)(1+\cos (\pi x)+d)}{2 \varepsilon}\left(1-e^{\frac{x d}{\varepsilon}}\right)}{\left(-1+e^{\frac{d}{2 \varepsilon}}\right)\left(-2-2 e^{\frac{d}{2 \varepsilon}}\right)+\frac{e^{1+\cos (\pi x)+d}}{4 \varepsilon}}+\sin (\pi x)^{2},
$$

where $d=\sqrt{5+2 \cos (\pi x)+\cos (\pi x)^{2}+4 \sin (\pi x / 2)}$. The $\varepsilon$-uniform convergence rates are calculated using the following expression:

$$
P^{N}=\ln \left(e^{N} / e^{2 N}\right) / \ln 2 .
$$

The error estimates are also denoted by

$$
e_{\varepsilon}^{N}=\left\|y^{\varepsilon, N}-u^{\varepsilon, N}\right\|_{\infty, \bar{\omega}_{N}}, \quad e^{N}=\max _{\varepsilon} e_{\varepsilon}^{N} .
$$

As presented in Table 1, when the $\varepsilon$ is small, the solution changes fastly in the boundary layer regions.

Table 1. Errors $e^{N}$ and rates of convergence $p^{N}$ for test problem.

\begin{tabular}{cccccccc}
\hline$\varepsilon \mid N$ & 16 & 32 & 64 & 128 & 256 & 512 & 1024 \\
\hline $2^{-15}$ & 0.1303665 & 0.0379424 & 0.0101458 & 0.0026100 & 0.0006577 & 0.0001630 & 0.0000395 \\
& 1.78 & 1.90 & 1.95 & 1.98 & 2.01 & 2.04 & \\
$2^{-16}$ & 0.1303729 & 0.0379483 & 0.0101519 & 0.0026147 & 0.0006609 & 0.0001650 & 0.0000406 \\
& 1.78 & 1.90 & 1.95 & 1.98 & 2.00 & 2.02 & \\
$2^{-17}$ & 0.1303761 & 0.0379513 & 0.0101549 & 0.0026171 & 0.0006625 & 0.0001660 & 0.0000412 \\
& 1.78 & 1.90 & 1.95 & 1.98 & 1.99 & 2.01 & \\
$2^{-18}$ & 0.1303777 & 0.0379528 & 0.0101564 & 0.0026183 & 0.0006633 & 0.0001666 & 0.0000415 \\
& 1.78 & 1.90 & 1.95 & 1.98 & 1.99 & 2.00 & \\
$2^{-19}$ & 0.1303785 & 0.0379535 & 0.0101572 & 0.0026189 & 0.0006637 & 0.0001668 & 0.0000417 \\
& 1.78 & 1.90 & 1.95 & 1.98 & 1.99 & 2.00 & \\
$2^{-20}$ & 0.1303789 & 0.0379539 & 0.0101575 & 0.0026192 & 0.0006639 & 0.0001669 & 0.0000418 \\
& 1.78 & 1.90 & 1.95 & 1.98 & 1.99 & 1.99 & \\
$2^{-21}$ & 0.1303791 & 0.0379541 & 0.0101577 & 0.0026193 & 0.0006640 & 0.0001670 & 0.0000418 \\
& 1.78 & 1.90 & 1.95 & 1.98 & 1.99 & 1.99 & \\
$e^{N}$ & 0.1303791 & 0.0379541 & 0.0101577 & 0.0026193 & 0.0006640 & 0.0001670 & 0.0000418 \\
$p^{N}$ & 1.78 & 1.90 & 1.95 & 1.98 & 1.99 & 1.99 & \\
\hline
\end{tabular}

When $N=32,64, \ldots, 1024$ takes increasing values, it is observed in table that the convergence rate $p^{N}$ is of the second-order. The exact solution and approximate solution curves are determined to be almost same, as presented in Figure 1. Therefore, it is possible to conclude that convergence is achieved. As indicated in Figure 2, errors in boundary layer regions with respect to the examination of the presented method for the problem (1.1)-(1.3), are maximum due to the irregularity caused by the sudden and rapid change of the solution in these regions around $x=0$ and $x=1$ for the different values of $\varepsilon$. Therefore, the numerical results indicated that the proposed scheme is working effectively. 


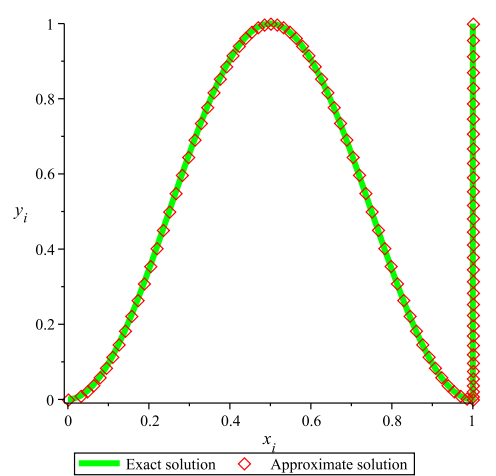

Figure 1. Comparison of the exact and approximate solutions of test problem for $N=128, \varepsilon=2^{-15}$.

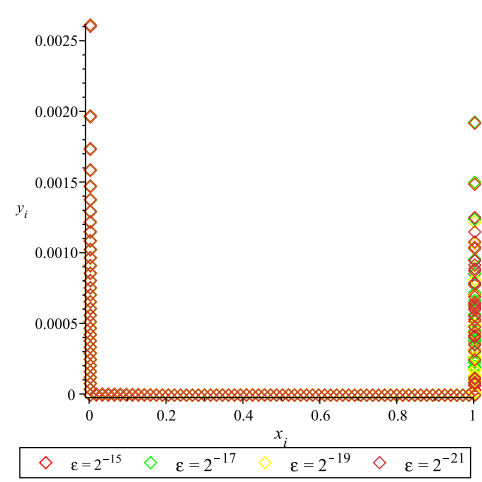

Figure 2. Error distribution of test problem for $N=128, \varepsilon=2^{-15}$, $\varepsilon=2^{-17}, \varepsilon=2^{-19}, \varepsilon=2^{-21}$.

\section{Conclusions}

In this study, we offered an effective finite difference method for solving secondorder linear singularly perturbed nonlocal boundary value problem. Uniform convergence in the second-order was proven with respect to the $\varepsilon$ - perturbation parameter in the discrete maximum norm of the difference scheme. As a result, it was possible to conclude that the finite difference method, taken into consideration for the solution of problems that are not easy to solve with every numerical method and that have both nonlocal and singular perturbation properties, was very effective and convenient on nonuniform meshes (Shishkin, Bakhvalov, Bakhvalov-Shishkin etc.). The present study findings demonstrate that it would be possible to conduct further studies on delayed and partial differential equations, which contain more complex nonlocal conditions. Furthermore, it could be suggested that a study on the increase in the convergence rate to three or higher orders would be possible.

\section{Acknowledgements}

The author is grateful to the referees and editor for their careful reading, valuable suggestions and helpful comments which helped improve the quality of this manuscript.

\section{References}

[1] G.M. Amiraliyev and M. Cakir. A uniformly convergent difference scheme for singularly perturbed problem with convective term and zeroth order reduced equation. Int. J. Appl. Math., 2(12):1407-1419, 2000.

[2] G.M. Amiraliyev and M. Cakir. Numerical solution of singularly perturbed problem with nonlocal boundary condition. Appl. Math. Mech., 23(7):755-764, 2002. https://doi.org/10.1007/BF02456971. 
[3] G.M. Amiraliyev and Y.D. Mamedov. Difference schemes on the uniform mesh for a singularly perturbed pseudo-parabolic equations. Turk. J. Math., 19(1995):207-222, 1995.

[4] D. Arslan. Finite difference method for solving singularly perturbed multi-point boundary value problem. J. Inst. Natural and Appl. Sci., 22(2):64-75, 2017.

[5] N.S. Bakhvalov. Towards optimization of methods for solving boundary value problems in the presence of a boundary layer. Zh. Vychisl. Mat. Mat. Fiz., 9(4):841-859, 1969. https://doi.org/10.1016/0041-5553(69)90038-X.

[6] A.V. Bitsadze and A.A. Samarskii. On some simpler generalization of linear elliptic boundary value problems. Doklady Akademii Nauk SSSR, 185:739-740, 1969.

[7] A. Bugajev and R. Čiegis. Comparison of adaptive meshes for a singularly perturbed reaction-diffusion problem. Math. Model. Anal., 17(5):732-748, 2012. https://doi.org/10.3846/13926292.2012.736416.

[8] M. Cakir. A numerical study on the difference solution of singularly perturbed semilinear problem with integral boundary condition. Math. Model. Anal., 21(5):644-658, 2016. https://doi.org/10.3846/13926292.2016.1201702.

[9] M. Cakir and G.M. Amiraliyev. Numerical solution of a singularly perturbed three-point boundary value problem. Int. J. Comput. Math., 84(10):1465-1481, 2007. https://doi.org/10.1080/00207160701296462.

[10] M. Cakir and D. Arslan. Finite difference method for nonlocal singularly perturbed problem. Int. J. of Modern Research Eng. Tech., 1(5):25-39, 2016. Available from Internet: www.ijmret.org.

[11] M. Cakir and D. Arslan. A numerical method for nonlinear singularly perturbed multi-point boundary value problem. J. Appl. Math. Phys., 4(6):1143-1156, 2016. https://doi.org/10.4236/jamp.2016.46119.

[12] M. Cakir and D. Arslan. Numerical solution of the nonlocal singularly perturbed problem. Int. J. of Modern Research Eng. Tech., 1(5):13-24, 2016. Available from Internet: www.ijmret.org.

[13] R. Čiegis. Numerical solution of a problem with small parameter for the highest derivative and a nonlocal condition. Liet. Mat. Rink., 28(1):144-152, 1988. https://doi.org/10.1007/BF00972255.

[14] R. Čiegis. On the difference schemes for problems with nonlocal boundary conditions. Informatica, 2(2):155-170, 1991.

[15] R. Čiegis, A. Štikonas, O. Štikonienè and O. Suboč. A monotonic finitedifference scheme for a parabolic problem with nonlocal conditions. Differ. Equ., 38(7):1027-1037, 2002. https://doi.org/10.1023/A:1021167932414.

[16] E. Cimen and G.M. Amiraliyev. A uniform convergent method for singularly perturbed nonlinear differential-difference equation. Journal of Informatics and Mathematical Sciences, 9(1):191-199, 2017. Available from Internet: www . projecteuclid.org.

[17] E. Cimen and M. Cakir. Numerical treatment of nonlocal boundary value problem with layer behaviour. Bull. Belg. Math. Soc. Simon Stevin, 24:339-352, 2017. https://doi.org/10.36045/bbms/1506477685.

[18] P.A. Farrel, A.F. Hegarty, J.J.H. Miller, E. ORiordan and G.I. Shishkin. Robust Computational Techniques for Boundary Layers. Chapman Hall/CRC, New York, 2000. https://doi.org/10.1201/9781482285727. 
[19] C.P. Gupta and S.I. Trofimchuk. A sharper condition for the solvability of a three-point second order boundary value problem. J. Math. Anal. Appl., 205:586-597, 1997. https://doi.org/10.1006/jmaa.1997.5252.

[20] D. Herceg and K. Surla. Solving a nonlocal singularly perturbed nonlocal problem by splines in tension. Univ u Novom Sadu Zb Rad Prirod-Mat Fak Ser Math., 21(2):119-132, 1991.

[21] T. Linss. An upwind difference scheme on a novel Shishkin-type mesh for a linear convection-diffusion problem. J. Comput. Appl. Mat., 110(1):93-104, 1999. https://doi.org/10.1016/S0377-0427(99)00198-3.

[22] T. Linss. Analysis of a Galerkin finite element method on a Bakhvalov-Shishkin mesh for a linear convection-diffusion problem. IMA J. Num. Anal., 20(4):621632, 2000. https://doi.org/10.1093/imanum/20.4.621.

[23] J.J.H. Miller, E.R. Doolan and W.H.A. Schilders. Uniform Numerical Methods for Problems with Initial and Boundary Layers. Boole Press, Dublin, 1980.

[24] J.J.H. Miller, E. ORiordan and G.I. Shishkin. Fitted Numerical Methods for Singular Perturbation Problems. World Scientic, Singapore, 1996. https://doi.org/10.1142/2933.

[25] A.H. Nayfeh. Introduction to Perturbation Techniques. Wiley, New York, 1993.

[26] R.E. OMalley. Singular Perturbation Methods for Ordinary Differential Equations. Springer Verlag, New York, 1991. https://doi.org/10.1007/978-1-46120977-5.

[27] M. Stynes, H.G. Roos and L. Tobiska. Robust Numerical Methods for Singularly Perturbed Differential Equations. Springer-Verlag, Berlin, 2008.

[28] Q. Zheng, X. Li and Y. Gao. Uniformly convergent hybrid schemes for solutions and derivatives in quasilinear singularly perturbed BVPs. Applied Numerical Mathematics, 91(2015):46-59, 2015. https://doi.org/10.1016/j.apnum.2014.12.010.

[29] Q. Zheng, X. Li and Y. Liu. Uniform second-order hybrid schemes on Bakhvalov-Shishkin mesh for quasi-linear convection-diffusion problems. Advanced Materials Research, 871(2014):135-140, 2014. https://doi.org/10.4028/www.scientific.net/AMR.871.135.

[30] P. Zhou, Y. Yin and Y. Yang. Finite element superconvergence on BakhvalovShishkin mesh for singularly perturbed problem. Journal on Numerical Methods and Computer Applications, 34(4):257-265, 2013. 